\title{
Best Proximity Points for Monotone Relatively Nonexpansive Mappings in Ordered Banach Spaces
}

\author{
Karim Chaira ${ }^{1, \dagger}$, Mustapha Kabil ${ }^{1, \dagger}{ }^{\dagger}$, Abdessamad Kamouss ${ }^{1, *,+} \oplus$ and Samih Lazaiz ${ }^{2, \dagger}$ \\ 1 Laboratory of Mathematics and Applications, Department of Mathematics, Faculty of Sciences and \\ Technologies Mohammedia, University Hassan II Casablanca, Casablanca 20000, Morocco; \\ chaira_karim@yahoo.fr (K.C.); kabilfstm@gmail.com (M.K.) \\ 2 Laboratory of Mathematical Analysis and Applications, Department of Mathematics, Faculty of Sciences \\ Dhar El Mahraz, University Sidi Mohamed Ben Abdellah, Fes 30050, Morocco; samih.lazaiz@gmail.com \\ * Correspondence: akamouss@gmail.com \\ $\dagger$ These authors contributed equally to this work.
}

Received: 23 September 2019; Accepted: 25 October 2019; Published: 1 November 2019

check for updates

\begin{abstract}
In this paper, we give sufficient conditions to ensure the existence of the best proximity point of monotone relatively nonexpansive mappings defined on partially ordered Banach spaces. An example is given to illustrate our results.
\end{abstract}

Keywords: best proximity point; fixed point; monotone mappings; relatively cyclic nonexpansive mappings; partially ordered Banach spaces

\section{Introduction}

Let $X$ be a Banach space and $(A, B)$ a pair of nonempty subsets of $X$. A cyclic mapping on $A \cup B$ is a mapping $T: A \cup B \rightarrow A \cup B$ such that $T(A) \subseteq B$ and $T(B) \subseteq A$. In case $A \cap B=\varnothing, T$ does not possess a fixed point, that is, a solution to the equation $T x=x$. Therefore, one can consider the following minimization problem:

$$
(P):\left\{\begin{array}{l}
\text { find }(x, y) \in A \times B \text { such that } \\
\|x-T x\|=\|y-T y\|=\operatorname{dist}(A, B) .
\end{array}\right.
$$

A point $x \in A \cup B$ is a best proximity point of $T$ if $x$ is a solution of the minimization problem $(P)$. The best proximity point notion can be seen as a generalization of fixed point notion since most fixed point theorems can be derived as corollaries of best proximity point theorems.

The first significant result of best proximity points was studied in [1], using the proximal normal structure, the authors proved that every cyclic relatively nonexpansive mapping from $A \cup B$ to itself has a best proximity point provided that $A$ and $B$ are weakly compact and convex. Furthermore, we find in [2] a similar result without invoking Zorn's lemma, i.e., without proximal normal structure. Recently, Chaira and Lazaiz [3] gave an extension of this last result in modular spaces. For a recent account of the theory we refer the reader to [4-6]. We can also find in ([7], pp. 27-31) an application of a best proximity point theorem to a system of differential equations.

On the other hand, the combination of metric fixed point theory and order theory allows Ran and Reurings in [8] to give a Banach Contraction Principle in partially ordered metric spaces. As consequence, they solved a matrix equation. Nieto and Rodríguez-López [9], extended the Ran-Reurings theorem in order to obtain a periodic solution for a first-order ordinary differential equation with periodic boundary conditions. 
Recently, many authors studied the existence of fixed points of monotone nonexpansive mappings defined on partially ordered Banach spaces (see for example [10-15]). Recall that a self mapping $T$ on $X$ is said to be monotone nonexpansive if $T$ is monotone and $\|T x-T y\| \leq\|x-y\|$, for every comparable elements $x$ and $y$. We should mention that monotone nonexpansive mappings may not be continuous. The interested reader can consult the book of Carl and Heikkilä [16] for many applications of fixed point results of monotone mappings.

In this work, motivated by the recent study of a fixed point for monotone mappings, we investigate the existence of the best proximity point of monotone relatively nonexpansive mappings in partially ordered Banach spaces.

\section{Preliminaries and Basic Results}

Let $(X,\|\|$.$) be a Banach space endowed with a partial order \preceq$. Throughout, we assume that the order intervals are closed and convex. Recall that an order interval is any of the subsets

$$
[a, \rightarrow)=\{x \in X ; a \preceq x\} \quad, \quad(\leftarrow, a]=\{x \in X ; x \preceq a\}
$$

for any $a \in X$. As a direct consequence of this, the subset

$$
[a, b]=\{x \in X ; a \preceq x \preceq b\}=[a, \rightarrow) \cap(\leftarrow, b]
$$

is also closed and convex for any $a, b \in X$.

We will say that $x, y \in X$ are comparable whenever $x \preceq y$ or $y \preceq x$. The linear structure of $X$ is assumed to be compatible with the order structure in the following sense:

(i) $\quad x \preceq y$ implies $x+z \preceq y+z$ for all $x, y, z \in X$;

(ii) $\quad x \preceq y$ implies $\alpha x \preceq \alpha y$ for all $x, y \in X$ and $\alpha \in \mathbb{R}^{+}$.

Let us recall the definition of a uniformly convex Banach space.

Definition 1. Let $(X,\|\|$.$) be a Banach space. We say that X$ is uniformly convex (in short, UC) if for every $\epsilon>0$ we have $\delta(\epsilon)>0$ such that

$$
\delta(\epsilon)=\inf \left\{1-\left\|\frac{x+y}{2}\right\| ;\|x\| \leq 1 ;\|y\| \leq 1 ;\|x-y\| \geq \epsilon\right\} .
$$

The function $\delta$ is known as the modulus of uniform convexity of X. Note that any UC Banach space is reflexive.

A sequence $\left\{x_{n}\right\}_{n \in \mathbb{N}}$ in a partially ordered set $(X, \preceq)$ is said to be

(i) monotone increasing if $x_{n} \preceq x_{n+1}$, for all $n \in \mathbb{N}$;

(ii) monotone decreasing if $x_{n+1} \preceq x_{n}$, for all $n \in \mathbb{N}$;

(iii) monotone sequence if it is either monotone increasing or decreasing.

The following technical lemmas will be useful to establish the main results.

Lemma 1. Let $X$ be a Banach space endowed with a partial order $\preceq$. Assume that $\left\{x_{n}\right\}$ and $\left\{y_{n}\right\}$ are two sequences on $X$ which are weakly convergent to $\bar{x}$ and $\bar{y}$ respectively and $x_{n} \preceq y_{n} \quad$ for any $n \in \mathbb{N}$, then

$$
\bar{x} \preceq \bar{y} .
$$

Proof. Note that the positive sequence $\left\{y_{n}-x_{n}\right\}_{n}$ converges weakly to $\bar{y}-\bar{x}$. Since closed convex subsets are also weakly closed, the positive cone is weakly closed and so we conclude that $\bar{y}-\bar{x}$ is positive. 
Lemma 2. [17] Let $\left\{x_{n}\right\}$ be a bounded monotone sequence in $X$, and assume that $X$ is reflexive. Then $\left\{x_{n}\right\}$ is weakly convergent.

Lemma 3. [18] Let C be a nonempty closed convex subset of a UC Banach space $(X,\|\|$.$) . Let \tau: C \rightarrow[0, \infty)$ be a type function, i.e., there exists a bounded sequence $\left\{x_{n}\right\} \in X$ such that

$$
\tau(x)=\limsup _{n \rightarrow \infty}\left\|x_{n}-x\right\|
$$

for every $x \in C$. Then $\tau$ has a unique minimum point $z \in C$ such that

$$
\tau(z)=\inf \{\tau(x) ; x \in C\}=\tau_{0}
$$

Moreover, if $\left\{z_{n}\right\}$ is a minimizing sequence in $C$, i.e., $\lim _{n \rightarrow \infty} \tau\left(z_{n}\right)=\tau_{0}$, then $\left\{z_{n}\right\}$ converges strongly to $z$.

The norm $\|\cdot\|$ of $X$ is said to be monotone if

$$
u \preceq v \preceq w \text { implies } \max \{\|w-v\|,\|v-u\|\} \leq\|w-u\|,
$$

for any $u, v, w \in X$. If the norm is monotone and $\left\{x_{n}\right\}$ is monotone increasing (respectively, decreasing), then the sequence $\left\{\left\|x_{n}-y\right\|\right\}$ is decreasing for any $y$ such that $x_{n} \preceq y$ (respectively, $y \preceq x_{n}$ ), for any $n \in \mathbb{N}$. In this case,

$$
\liminf _{n \rightarrow \infty}\left\|x_{n}-y\right\|=\lim _{n \rightarrow \infty}\left\|x_{n}-y\right\|=\inf _{n \in \mathbb{N}}\left\|x_{n}-y\right\|
$$

Recall that a mapping $T: X \rightarrow X$ is said to be

(i) monotone increasing if $x \preceq y$ implies $T(x) \preceq T(y)$, for all $x, y \in X$;

(ii) monotone decreasing if $x \preceq y$ implies $T(y) \preceq T(x)$, for all $x, y \in X$.

We conclude this section by extending the concept of relatively cyclic nonexpansive mapping to monotone relatively cyclic nonexpansive mapping as follows:

Definition 2. Let $(X,\|\|,. \preceq)$ be a Banach space endowed with a partially order and $(A, B)$ a pair of nonempty subset of $X$. The mapping $T: A \cup B \rightarrow A \cup B$ is said to be monotone increasing (respectively decreasing) relatively cyclic nonexpansive if

1. $T(A) \subseteq B$ and $T(B) \subseteq A$,

2. $T$ is monotone increasing (respectively decreasing),

3. $\|T x-T y\| \leq\|x-y\|$, whenever $x \in A$ and $y \in B$ are comparables.

\section{Main Result}

Throughout we assumed that $(X,\|\cdot\|, \preceq)$ is a Banach space endowed with a partial order for which order intervals are convex and closed and the linear structure of $X$ is assumed to be compatible with the order structure.

The following result gives sufficient conditions to obtain a fixed point theorem for a monotone increasing relatively cyclic nonexpansive mapping.

Theorem 1. Let $(A, B)$ be a nonempty bounded closed convex pair in a partially ordered Banach space $(X,\|\cdot\|, \preceq$ ). Assume that $(X,\|\|$.$) is UC. Let T: A \cup B \rightarrow A \cup B$ be a monotone increasing relatively cyclic nonexpansive mapping such that $x_{0} \preceq T x_{0}$ for some $x_{0} \in A$, then $A \cap B \neq \varnothing$ and there exists $a^{*} \in A \cap B$ such that $T a^{*}=a^{*}$. 
Proof. We assume that $x_{0} \preceq T x_{0}$ and we define the sequence $\left\{x_{n}\right\}$ by $x_{n+1}=T x_{n}$ for all $n \geq 0$. By using the monotonicity of $T$ we get

$$
x_{0} \preceq x_{1} \preceq \cdots \preceq x_{n} \preceq x_{n+1} \preceq \cdots
$$

Since $A$ and $B$ are bounded and closed, the sequence $\left\{x_{n}\right\}$ is bounded increasing in the reflexive space $X$. By Lemma 2,

$$
x_{2 n} \stackrel{w}{\longrightarrow} \bar{x}_{1} \in A \quad \text { and } \quad x_{2 n+1} \stackrel{w}{\longrightarrow} \bar{x}_{2} \in B .
$$

By uniqueness of the weak limit, $\bar{x}=\bar{x}_{1}=\bar{x}_{2}$. We claim that $A \cap B \neq \varnothing$.

Let $K=\left\{x \in A \cap B, \quad x_{n} \preceq x \quad\right.$ for all $\left.n \in \mathbb{N}\right\}$. It is clear that $K$ is nonempty, closed and convex set. Since $\left\{x_{n}\right\}$ is a bounded sequence in $X$, we can define the type function as follows

$$
\tau(x)=\limsup _{n \rightarrow \infty}\left\|x_{n}-x\right\|
$$

for any $x \in K$. From Lemma 3 , it follows that there exists a unique $a^{*} \in K$ such that

$$
\tau\left(a^{*}\right)=\inf _{x \in K} \tau(x)
$$

We have

$$
\tau\left(T a^{*}\right)=\limsup _{n \rightarrow \infty}\left\|x_{n}-T a^{*}\right\|=\limsup _{n \rightarrow \infty}\left\|T x_{n-1}-T a^{*}\right\| .
$$

Since $x_{n-1} \preceq a^{*}$ and $T$ is monotone relatively cyclic nonexpansive mapping,

$$
\tau\left(T a^{*}\right) \leq \limsup _{n \rightarrow \infty}\left\|x_{n-1}-a^{*}\right\|=\tau\left(a^{*}\right)
$$

Hence, $\tau\left(T a^{*}\right)=\tau\left(a^{*}\right)$. Thus $T a^{*}=a^{*}$, which completes the proof.

If $B=A$, we get the next result for a monotone nonexpansive mapping.

Corollary 1. Let $A$ be a nonempty bounded closed convex set in a partially ordered Banach space $(X,\|\cdot\|, \preceq)$. Let $T: A \rightarrow A$ be a monotone increasing nonexpansive mapping. Assume that $(X,\|\|$.$) is UC and there exists$ $x_{0} \in A$ such that $x_{0} \preceq T x_{0}$, then there exists $a^{*} \in A$ such that $T a^{*}=a^{*}$.

Now let $\left(\mathcal{A}_{0}^{\preceq}, \mathcal{B}_{0}^{\preceq}\right)$ denotes the pair obtained from $(A, B)$ upon setting

$$
\begin{aligned}
& \mathcal{A}_{0}^{\preceq}=\{x \in A ;\|x-y\|=\operatorname{dist}(A, B) \text { for some } y \in B \cap[x, \rightarrow)\} \\
& \mathcal{B}_{0}^{\preceq}=\{y \in B ;\|y-x\|=\operatorname{dist}(A, B) \text { for some } x \in A \cap(\leftarrow, y]\} .
\end{aligned}
$$

Lemma 4. Let $(A, B)$ be a nonempty bounded closed convex pair in a partially ordered reflexive Banach space $(X,\|\cdot\|, \preceq)$. Then,

(i) $\mathcal{A}_{0}^{\preceq} \neq \varnothing$ if and only if $\mathcal{B}_{0}^{\preceq} \neq \varnothing$;

(ii) $\operatorname{dist}\left(\mathcal{A}_{0}^{\preceq}, \mathcal{B}_{0}^{\preceq}\right)=\operatorname{dist}(A, B)$;

(iii) $\left(\mathcal{A}_{0}^{\preceq}, \mathcal{B}_{0}^{\preceq}\right)$ is a closed pair;

(iv) $\left(\mathcal{A}_{0}^{\preceq}, \mathcal{B}_{0}^{\preceq}\right)$ is a convex pair.

Proof. Using the definitions of $\mathcal{A}_{0}^{\preceq}$ and $\mathcal{B}_{0}^{\preceq}$, we can easily derive $(i)$ and (ii).

(iii) Let $\left\{x_{n}\right\} \subset \mathcal{A}_{0}^{\preceq}$ be a sequence which converges to some $\bar{x}$ in $A$. Then there exists a sequence $\left\{y_{n}\right\} \subset B$ such that

$$
\left\|x_{n}-y_{n}\right\|=\operatorname{dist}(A, B) \text { and } x_{n} \preceq y_{n}
$$


Since $B$ is closed and bounded in a reflexive Banach space, there exists a subsequence $\left\{y_{\varphi(n)}\right\}$ of $\left\{y_{n}\right\}$ such that $y_{\varphi(n)} \stackrel{w}{\longrightarrow} \bar{y} \in B$. From Lemma 1 , it follows that $\bar{x} \preceq \bar{y}$. On the other hand,

$$
\|\bar{x}-\bar{y}\| \leq \liminf _{n \rightarrow \infty}\left\|x_{\varphi(n)}-y_{\varphi(n)}\right\|=\operatorname{dist}(A, B) .
$$

Therefore, we have $\bar{x} \in \mathcal{A}_{0}^{\preceq}$, and hence, $\mathcal{A}_{0}^{\preceq}$ is closed. By the same arguments we get that $\mathcal{B}_{0}^{\preceq}$ is also closed.

(iv) Let $x$ and $x^{\prime}$ in $\mathcal{A}_{0}^{\prec}$. Then there exist $y$ and $y^{\prime}$ in $B$ such that

$$
\left\{\begin{array}{c}
\|x-y\|=\operatorname{dist}(A, B) \text { and } x \preceq y \\
\left\|x^{\prime}-y^{\prime}\right\|=\operatorname{dist}(A, B) \text { and } x^{\prime} \preceq y^{\prime} .
\end{array}\right.
$$

By using the fact that the linear structure of $X$ is compatible with the order structure, we get for any $t \in[0,1]$

$$
\begin{aligned}
\left\|t x+(1-t) x^{\prime}-t y-(1-t) y^{\prime}\right\| & =\left\|t(x-y)+(1-t)\left(x^{\prime}-y^{\prime}\right)\right\| \\
& \leq t\|x-y\|+(1-t)\left\|x^{\prime}-y^{\prime}\right\| \\
& =\operatorname{dist}(A, B)
\end{aligned}
$$

This implies that $t x+(1-t) x^{\prime} \in \mathcal{A}_{0}^{\prec}$. It follows that $\mathcal{A}_{0}^{\prec}$ is convex, as claimed. Similarly we prove that $\mathcal{B}_{0}^{\prec}$ is also convex.

Remark 1. Note that if $T$ is a monotone decreasing relatively cyclic nonexpansive mapping, we have $T\left(\mathcal{A}_{0}^{\prec}\right) \subset$ $\mathcal{B}_{0}^{\prec}$ and $T\left(\mathcal{B}_{0}^{\prec}\right) \subset \mathcal{A}_{0}^{\prec}$. Indeed, let $x \in \mathcal{A}_{0}^{\prec}$ then there exists $y \in B$ such that

$$
\|x-y\|=\operatorname{dist}(A, B) \quad \text { and } x \preceq y .
$$

Thus,

$$
\|T x-T y\| \leq\|x-y\|=\operatorname{dist}(A, B) \text { and } T y \preceq T x .
$$

This implies $T x \in \mathcal{B}_{0}^{\preceq}$. Consequently $T\left(\mathcal{A}_{0}^{\prec}\right) \subset \mathcal{B}_{0}^{\prec}$.

For the sake of simplicity, we use the following notation

$$
\mathcal{A}_{T}=\left\{\left(x_{0}, x_{0}^{\prime}\right) \in A \times A ; x_{0} \preceq T x_{0}^{\prime} ;\left\|x_{0}-T x_{0}^{\prime}\right\|=\operatorname{dist}(A, B)\right\} .
$$

The next lemma gives sufficient conditions such that $\mathcal{A}_{T}$ is nonempty.

Lemma 5. Let $(A, B)$ be a nonempty bounded closed convex pair in a partially ordered Banach space $(X,\|\cdot\|, \preceq)$ such that $\mathcal{A}_{0}^{\prec}$ is nonempty. Let $T: A \cup B \rightarrow A \cup B$ be a monotone relatively cyclic nonexpansive mapping. Then $\mathcal{A}_{T}$ is nonempty.

Proof. Suppose that $T$ is a monotone decreasing relatively cyclic nonexpansive mapping. Since $\mathcal{A}_{0}^{\prec} \neq$ $\varnothing$, we can find a $x_{0}^{\prime}$ in $\mathcal{A}_{0}^{\prec}$ such that there exists an $y \in B \cap\left[x_{0}^{\prime}, \rightarrow\right)$ satisfying $\left\|x_{0}^{\prime}-y\right\|=\operatorname{dist}(A, B)$.

Since $x_{0}^{\prime} \preceq y$ and $T$ is monotone decreasing relatively cyclic nonexpansive mapping, $T y \preceq T x_{0}^{\prime}$ and $\left\|T x_{0}^{\prime}-T y\right\| \leq\left\|x_{0}^{\prime}-y\right\|=\operatorname{dist}(A, B)$, give that $T x_{0}^{\prime} \in \mathcal{B}_{0}^{-}$.

Next, for $T x_{0}^{\prime}$ there exists an element $x_{0} \in \mathcal{A}_{0}^{\prec}$ such that

$$
x_{0} \preceq T x_{0}^{\prime} \quad \text { and } \quad\left\|x_{0}-T x_{0}^{\prime}\right\|=\operatorname{dist}(A, B) .
$$


Now, suppose that $T$ is a monotone increasing relatively cyclic nonexpansive mapping. Since $\mathcal{A}_{0}^{\prec} \neq \varnothing$, we can find a $x$ in $\mathcal{A}_{0}^{\preceq}$ such that there exists an $y \in \mathcal{B}_{0}^{\prec}$ satisfying $x \preceq y$ and $\|x-y\|=\operatorname{dist}(A, B)$.

Since $T$ is monotone increasing, $T x \preceq T y$ and

$$
\left\|T^{2} x-T^{2} y\right\| \leq\|T x-T y\| \leq\|x-y\|=\operatorname{dist}(A, B) .
$$

Take $x_{0}=T^{2} x \in A$ and $x_{0}^{\prime}=T y \in A$. We have clearly,

$$
x_{0} \preceq T x_{0}^{\prime} \quad \text { and } \quad\left\|x_{0}-T x_{0}^{\prime}\right\|=\operatorname{dist}(A, B) .
$$

Thus $\mathcal{A}_{T} \neq \varnothing$.

In the following, we give a best proximity result for monotone increasing relatively cyclic nonexpansive mapping.

Theorem 2. Let $(X,\|\|,. \preceq)$ be a partially ordered Banach space. Assume that $(X,\|\|$.$) is UC. Let (A, B)$ be a nonempty bounded closed convex pair in $X$. Let $T: A \cup B \rightarrow A \cup B$ be a monotone increasing relatively cyclic nonexpansive mapping. Assume that $T$ is weakly sequentially continuous, the norm $\|$.$\| of X$ is monotone and there exists $\left(x_{0}, x_{0}^{\prime}\right) \in \mathcal{A}_{T}$ such that $x_{0} \preceq x_{0}^{\prime} \preceq T^{2} x_{0}$ then there exist $\bar{x} \in A$ and $\bar{y} \in B$ such that $\|\bar{x}-T \bar{x}\|=\|\bar{y}-T \bar{y}\|=\operatorname{dist}(A, B)$.

Proof. Suppose that there exists $\left(x_{0}, x_{0}^{\prime}\right) \in A \times A$ such that

$$
\left\|x_{0}-T x_{0}^{\prime}\right\|=\operatorname{dist}(A, B) \quad \text { and } \quad x_{0} \preceq x_{0}^{\prime} \preceq T^{2} x_{0} .
$$

Let $\left\{x_{n}\right\}$ and $\left\{y_{n}\right\}$ be two sequences defined as follows:

$$
\left\{\begin{array}{l}
x_{n}=T^{2 n} x_{0} \\
y_{n}=T^{2 n+1} x_{0}^{\prime}
\end{array} \quad \text { for all } n \in \mathbb{N} .\right.
$$

Note that, since $x_{0} \preceq T x_{0}^{\prime}$ we get $T^{2 n} x_{0} \preceq T^{2 n+1} x_{0}^{\prime}$ for all $n \geq 0$, that is, $x_{n} \preceq y_{n}$ for all $n \geq 0$.

Since $T$ is monotone increasing relatively cyclic nonexpansive mapping, we get

$$
\left\|x_{n}-y_{n}\right\|=\left\|T^{2 n} x_{0}-T^{2 n+1} x_{0}^{\prime}\right\| \leq\left\|x_{0}-T x_{0}^{\prime}\right\|=\operatorname{dist}(A, B),
$$

that is, $\left\|x_{n}-y_{n}\right\|=\operatorname{dist}(A, B)$, for all $n \in \mathbb{N}$.

Since $x_{0} \preceq T^{2} x_{0}, x_{1}=T^{2} x_{0} \preceq T^{4} x_{0}=x_{2}$ and by induction on $n$, we can get

$$
x_{n} \preceq x_{n+1} \text { for all } n \in \mathbb{N} \text {. }
$$

In the same manner, we get

$$
y_{n} \preceq y_{n+1} \quad \text { for all } n \in \mathbb{N} \text {. }
$$

Since $\left\{x_{n}\right\}$ and $\left\{y_{n}\right\}$ are bounded increasing sequences in reflexive space, we get from Lemma 2, $x_{n} \stackrel{w}{\longrightarrow} \bar{x}$ and $y_{n} \stackrel{w}{\longrightarrow} \bar{y}$.

Note that $\bar{x}=\sup \left\{x_{n} ; n \in \mathbb{N}\right\}$ and $\bar{y}=\sup \left\{y_{n} ; n \in \mathbb{N}\right\}$.

Let $K=\left\{y \in B ; y_{n} \preceq y, \quad\right.$ for any $\left.n \in \mathbb{N}\right\}$ and define the type function $\tau: K \rightarrow[0, \infty)$ generated by the sequence $\left\{x_{n}\right\}$, that is,

$$
\tau(y)=\limsup _{n \rightarrow \infty}\left\|x_{n}-y\right\|,
$$


for $y \in K$. Using the fact that $\tau$ is increasing function, we get

$$
\tau(\bar{y})=\inf _{y \in K} \tau(y) .
$$

Indeed, let $z_{1}, z_{2} \in K$ such that $z_{1} \preceq z_{2}$ then for all $n \in \mathbb{N}$ we have

$$
x_{n} \preceq y_{n} \preceq z_{1} \preceq z_{2} .
$$

Using the fact that the norm $\|\cdot\|$ is monotone, we get

$$
\left\|x_{n}-z_{1}\right\| \leq\left\|x_{n}-z_{2}\right\|,
$$

hence,

$$
\tau\left(z_{1}\right) \leq \tau\left(z_{2}\right) .
$$

From Lemma 3, it follows that there exists a unique $b^{*} \in K$ such that :

$$
\tau\left(b^{*}\right)=\inf _{y \in K} \tau(y) .
$$

Since $\bar{y}=\sup \left\{y_{n} ; n \in \mathbb{N}\right\}$ and $b^{*} \in K, \bar{y} \preceq b^{*}$, that is, $\tau(\bar{y}) \preceq \tau\left(b^{*}\right)$.

Thus, $\tau(\bar{y})=\tau\left(b^{*}\right)$, i.e., $\bar{y}=b^{*}$.

We have also

$$
\begin{aligned}
\tau\left(T^{2} \bar{y}\right) & =\limsup _{n \rightarrow \infty}\left\|x_{n}-T^{2} \bar{y}\right\| \\
& =\limsup _{n \rightarrow \infty}\left\|T^{2} x_{n-1}-T^{2} \bar{y}\right\| \\
& \leq \limsup _{n \rightarrow \infty}\left\|x_{n-1}-\bar{y}\right\| \\
& =\tau(\bar{y}),
\end{aligned}
$$

hence, $T^{2} \bar{y}=\bar{y}$.

Furthermore, $T$ is weakly sequentially continuous then $T x_{n} \stackrel{w}{\longrightarrow} T \bar{x}$ and $T y_{n} \stackrel{w}{\longrightarrow} T \bar{y}$. By the lower semi continuity of the norm, we get

$$
\|\bar{x}-\bar{y}\| \leq \liminf _{n \rightarrow \infty}\left\|x_{n}-y_{n}\right\|=\operatorname{dist}(A, B) .
$$

Let $\left\{x_{n}^{\prime}\right\}$ be a sequence defined by $x_{n}^{\prime}=T^{2 n} x_{0}^{\prime}$, for all $n \in \mathbb{N}$. We have

$$
y_{n}=T^{2 n+1} x_{0}^{\prime}=T\left(T^{2 n} x_{0}^{\prime}\right)=T x_{n}^{\prime} .
$$

Since $x_{0}^{\prime} \preceq T^{2} x_{0}^{\prime}, T^{2 n} x_{0}^{\prime} \preceq T^{2 n+2} x_{0}^{\prime}$, that is, $x_{n}^{\prime} \preceq x_{n+1}^{\prime}$, for all $n \in \mathbb{N}$. Since $\left\{x_{n}^{\prime}\right\}$ is bounded increasing sequence in reflexive space, we get by using Lemma $2 x_{n}^{\prime} \stackrel{w}{\longrightarrow} \bar{x}^{\prime}$. Since $T$ is weakly sequentially continuous, $y_{n}=T x_{n}^{\prime} \stackrel{w}{\longrightarrow} T \bar{x}^{\prime}$. By the uniqueness of the limit, $T \bar{x}^{\prime}=\bar{y}$, that is,

$$
\left\|\bar{x}-\bar{x}^{\prime}\right\|=\operatorname{dist}(A, B) \text {. }
$$

Note that $x_{0} \preceq x_{0}^{\prime} \preceq T^{2} x_{0} \preceq T^{2} x_{0}^{\prime}$, that is, $x_{0} \preceq x_{0}^{\prime} \preceq x_{1} \preceq x_{1}^{\prime}$. Then, by induction on $n$, we can get

$$
x_{n} \preceq x_{n}^{\prime} \preceq x_{n+1} \preceq x_{n+1}^{\prime} .
$$


Define the sequence $\left\{z_{n}\right\}$ as follows

$$
z_{n}=\left\{\begin{array}{cc}
x_{n} & \text { if } n \text { is even, } \\
x_{n-1}^{\prime} & \text { if } n \text { is odd }
\end{array}\right.
$$

Since $\left\{z_{n}\right\}$ is bounded increasing sequence in reflexive space, by using Lemma 2, we get $z_{n} \stackrel{w}{\longrightarrow} \bar{z}$. In particular, the subsequences $\left\{z_{2 n}\right\}$ and $\left\{z_{2 n+1}\right\}$ also converge to $\bar{z}$, that is, $\bar{z}=\bar{x}=\bar{x}^{\prime}$. Thus, by using (3) we get $\|\bar{x}-T \bar{x}\|=\operatorname{dist}(A, B)$.

In the following, we give a best proximity result for monotone decreasing relatively cyclic nonexpansive mapping without assuming the monotonicity of the norm $\|$.$\| .$

Theorem 3. Let $(A, B)$ be a nonempty bounded closed convex pair in a partially ordered Banach space $(X,\|\|,. \preceq)$. Let $T: A \cup B \rightarrow A \cup B$ be a monotone decreasing relatively cyclic nonexpansive mapping. Assume that $(X,\|\cdot\|)$ is $U C, T$ is weakly sequentially continuous and there exists $\left(x_{0}, x_{0}^{\prime}\right) \in \mathcal{A}_{T}$ such that $x_{0}^{\prime} \preceq x_{0} \preceq T^{2} x_{0}^{\prime}$, then there exists $(\bar{x}, \bar{y}) \in A \times B$ such that

$$
\|\bar{x}-T \bar{x}\|=\|\bar{y}-T \bar{y}\|=\operatorname{dist}(A, B) .
$$

Proof. Let $\left(x_{0}, x_{0}^{\prime}\right) \in \mathcal{A}_{T}$ such that

$$
x_{0}^{\prime} \preceq x_{0} \preceq T^{2} x_{0}^{\prime} .
$$

If $A \cap B \neq \varnothing$ then $x_{0}=T x_{0}^{\prime}$ by Lemma 5. Since $x_{0}^{\prime} \preceq x_{0} \preceq T^{2} x_{0}^{\prime}$ and $T$ is decreasing, we get $x_{0} \preceq T x_{0}$ and $T x_{0} \preceq T x_{0}^{\prime}=x_{0}$. Thus, $T x_{0}=x_{0}$.

If $A \cap B=\varnothing$, then we consider the sequences $\left\{x_{n}\right\}$ and $\left\{z_{n}\right\} \subset A$ defined by

$$
\left\{\begin{array}{l}
z_{0}=x_{0}^{\prime} \\
x_{n}=T^{2 n} x_{0} \quad \text { for all } n \in \mathbb{N}^{*} . \\
z_{n}=T^{2 n} x_{0}^{\prime}
\end{array}\right.
$$

Since $x_{0}^{\prime} \preceq x_{0} \preceq T^{2} x_{0}^{\prime}=z_{1}$ and $T^{2}$ is a monotone increasing mapping, by induction on $n$, we get $T^{2 n} x_{0}^{\prime} \preceq T^{2 n} x_{0} \preceq T^{2 n+2} x_{0}^{\prime}$, which implies

$$
z_{n} \preceq x_{n} \preceq z_{n+1}
$$

for all $n \geq 0$. Also, since $x_{0} \preceq T x_{0}^{\prime}=T z_{0}$ and $T^{2}$ is a monotone increasing mapping, by induction on $n$, we get $T^{2 n} x_{0} \preceq T\left(T^{2 n} x_{0}^{\prime}\right)$, which implies

$$
x_{n} \preceq T z_{n},
$$

for all $n \geq 0$. The sequences $\left\{x_{n}\right\}$ and $\left\{z_{n}\right\}$ are increasing. Indeed, $x_{0} \preceq T^{2} x_{0}^{\prime} \preceq T^{2} x_{0}$ implies by induction on $n$ that $T^{2 n} x_{0} \preceq T^{2 n+2} x_{0}$. Thus,

$$
x_{n} \preceq x_{n+1},
$$

for all $n \in \mathbb{N}$. Since $\left\{x_{n}\right\}$ and $\left\{z_{n}\right\}$ are bounded increasing sequences in a reflexive space, we get by Lemma $2, x_{n} \stackrel{w}{\longrightarrow} \bar{x}$ and $z_{n} \stackrel{w}{\longrightarrow} \bar{z}$. Using the fact that $T$ is weakly sequentially continuous we conclude that $T z_{n} \stackrel{w}{\longrightarrow} T \bar{z}$. 
Since $T$ is relatively cyclic nonexpansive mapping, we get

$$
\begin{aligned}
\left\|x_{n}-T z_{n}\right\| & =\left\|T^{2} x_{n-1}-T^{3} z_{n-1}\right\| \\
& \leq\left\|T x_{n-1}-T^{2} z_{n-1}\right\| \\
& \leq\left\|x_{n-1}-T z_{n-1}\right\|,
\end{aligned}
$$

for all $n$ in $\mathbb{N}^{*}$. By induction on $n$, we prove that

$$
\left\|x_{n}-T z_{n}\right\| \leq\left\|x_{0}-T x_{0}^{\prime}\right\|=\operatorname{dist}(A, B),
$$

for all $n \in \mathbb{N}$. By the lower semi continuity of the norm, we get

$$
\|\bar{x}-T \bar{z}\| \leq \liminf _{n \rightarrow \infty}\left\|x_{n}-T z_{n}\right\|=\operatorname{dist}(A, B) .
$$

It follows from the Lemma 1 and the inequality (4) that $\bar{z} \preceq \bar{x} \preceq \bar{z}$, and hence, $\bar{z}=\bar{x}$. Finally, by Equation (6) it follows that

$$
\|\bar{x}-T \bar{x}\|=\operatorname{dist}(A, B) .
$$

Let $\bar{y}=T \bar{x}$, then by inequality (5) and Lemma 1 we have $\bar{x} \preceq \bar{y}$ and

$$
\|\bar{y}-T \bar{y}\|=\|T \bar{x}-T \bar{y}\| \leq\|\bar{x}-\bar{y}\|=\operatorname{dist}(A, B) .
$$

So the proof is complete.

We claim that $T^{2} \bar{x}=\bar{x}$ and $T^{2} \bar{y}=\bar{y}$. Indeed, since $x_{n+1}=T^{2} x_{n} \stackrel{w}{\longrightarrow} \bar{x}$ and $x_{n+1}=T^{2} x_{n} \stackrel{w}{\longrightarrow} T^{2} \bar{x}$, the uniqueness of the weak limit implies that $T^{2} \bar{x}=\bar{x}$. Furthermore, $T \bar{x}=\bar{y}$ then

$$
T^{2} \bar{x}=\bar{x} \Longrightarrow T\left(T^{2} \bar{x}\right)=T \bar{x} \Longrightarrow T^{2}(T \bar{x})=\bar{y} \Longrightarrow T^{2} \bar{y}=\bar{y} .
$$

The following example illustrates Theorem 3.

Example 1. Consider $X=\mathbb{R}^{2}$ with usual norm and the partially order defined by:

$$
(a, b) \preceq(c, d) \quad \text { iff } \quad(a \leq c \quad \text { and } \quad b \leq d),
$$

for any $(a, b),(c, d)$ in $\mathbb{R}^{2}$. Suppose that

$$
\begin{gathered}
A=\left\{(x, 0) \in \mathbb{R}^{2} ; x \in[0,2]\right\} \text { and } \\
B=\left\{(x, 1) \in \mathbb{R}^{2} ; x \in[2,4]\right\},
\end{gathered}
$$

we can show that $\operatorname{dist}(A, B)=1, \mathcal{A}_{0}^{\prec}=\{(2,0)\}$ and $\mathcal{B}_{0}^{\prec}=\{(2,1)\}$. Suppose that a mapping $T: A \cup B \rightarrow$ $A \cup B$ is defined as follows

$$
\left\{\begin{array}{l}
T(x, 0)=(2,1) ; \quad \text { for all } \quad(x, 0) \in A \\
T(x, 1)=(4-x, 0) ; \quad \text { for all } \quad(x, 1) \in B
\end{array}\right.
$$


We have $T(A) \subset B, T(B) \subset A$ and $T$ is a decreasing mapping. Also, for any $\left((x, 0),\left(x^{\prime}, 1\right)\right) \in A \times B$ we have $(x, 0) \preceq\left(x^{\prime}, 1\right)$ and

$$
\left\{\begin{aligned}
\left\|T(x, 0)-T\left(x^{\prime}, 1\right)\right\| & =\left\|(2,1)-\left(4-x^{\prime}, 0\right)\right\| \\
& =\sqrt{\left(x^{\prime}-2\right)^{2}+1} \\
\left\|(x, 0)-\left(x^{\prime}, 1\right)\right\| & =\left\|\left(x^{\prime}-x, 1\right)\right\| \\
& =\sqrt{\left(x^{\prime}-x\right)^{2}+1}
\end{aligned}\right.
$$

thus, $\left\|T(x, 0)-T\left(x^{\prime}, 1\right)\right\| \leq\left\|(x, 0)-\left(x^{\prime}, 1\right)\right\|$. Then $T$ is a monotone decreasing relatively cyclic nonexpansive mapping.

If we choose $x_{0}^{\prime}=(0,0)$ and $x_{0}=(2,0)$ in $A$ we get

$$
x_{0} \preceq T x_{0}^{\prime}, \quad\left\|x_{0}-T x_{0}^{\prime}\right\|=\operatorname{dist}(A, B) \quad \text { and } \quad x_{0}^{\prime} \preceq x_{0} \preceq T^{2} x_{0}^{\prime} .
$$

Then there exist $\bar{x}=(2,0) \in A$ and $\bar{y}=(2,1) \in B$ such that $T^{2} \bar{x}=\bar{x}, T^{2} \bar{y}=\bar{y}$ and

$$
\|\bar{x}-T \bar{x}\|=\|\bar{y}-T \bar{y}\|=\operatorname{dist}(A, B) .
$$

Author Contributions: The authors contributed equally to this work.

Funding: This research received no external funding.

Conflicts of Interest: The authors declare no conflict of interest.

\section{References}

1. Eldred, A.; Kirk, W.; Veeramani, P. Proximal normal structure and relatively nonexpansive mappings. Studia Math. 2005, 3, 283-293. [CrossRef]

2. Sankar Raj, V.; Veeramani, P. Best proximity pair theorems for relatively nonexpansive mappings. Appl. Gen. Topol. 2009, 10, 21-28. [CrossRef]

3. Chaira, K.; Lazaiz, S. Best proximity point theorems for cyclic relatively nonexpansive mappings in modular spaces. Abstr. Appl. Anal. 2018, 2018, 8084712. [CrossRef]

4. Espinola, R. A new approach to relatively nonexpansive mappings. Proc. Am. Math. Soc. 2008, 136, 1987-1995. [CrossRef]

5. Ggabeleh, M. Cyclic relatively nonexpansive mappings in convex metric spaces. Miskolc Math. Notes 2015, 16, 133-144. [CrossRef]

6. Chaira, K.; Lazaiz, S. Best proximity pair and fixed point results for noncyclic mappings in modular spaces. Arab J. Math. Sci. 2018, 24, 147-165. [CrossRef]

7. Veeramani, P.; Rajesh, S. Best proximity points. In Nonlinear Analysis: Approximation Theory, Optimization and Applications, 3rd ed.; Ansari, Q.H., Ed.; Springer Science + Business Media: New York, NY, USA, 2014; pp. 1-32, ISBN 978-81-322-1882-1.

8. Ran, A.C.M.; Reurings, M.C.B. A fixed point theorem in partially ordered sets and some applications to matrix equations. Proc. Am. Math. Soc. 2004, 132, 1435-1443. [CrossRef]

9. Nieto, J.J.; Rodríguez-López, R. Contractive mapping theorems in partially ordered sets and applications to ordinary differential equations. Order 2005, 22, 223-239. [CrossRef]

10. Zhang, X. Fixed point theorems of multivalued monotone mappings in ordered metric spaces. Appl. Math. Lett. 2010, 23, 235-240. [CrossRef]

11. Bachar, M.; Khamsi, M.A. Fixed points of monotone mappings and application to integral equations. Fixed Point Theory Appl. 2015, 2015, 110. [CrossRef]

12. Pandey, R.; Pant, R.; Al-Rawashdeh, A. Fixed point results for a class of monotone nonexpansive type mappings in hyperbolic spaces. J. Funct. Spaces 2018, 2018, 5850181. [CrossRef] 
13. Alfuraidan, M.R.; Jorquera, E.D.; Khamsi, M.A. Fixed point theorems for monotone Caristi inward mappings. Numer. Funct. Anal. Optim. 2018, 39, 1092-1101. [CrossRef]

14. Van Dung, N.; Hieu, N.T.; Radojević, S. Fixed point theorems for g-monotone maps on partially ordered S-metric spaces. Filomat 2014, 28, 1885-1898. [CrossRef]

15. Dehaish, B.A.B.; Khamsi, M.A. Mann iteration process for monotone nonexpansive mappings. Fixed Point Theory Appl. 2015, 2015, 177. [CrossRef]

16. Carl, S.; Heikkilä, S. Fixed Point Theory in Ordered Sets and Applications: From Differential and Integral Equations to Game Theory, 1st ed.; Springer Science + Business Media: New York, NY, USA, 2011; ISBN 978-1-4419-7584-3.

17. Alfuraidan, M.R.; Khamsi, M.A. Fibonacci Mann iteration for monotone asymptotically nonexpansive mappings. Bull. Aust. Math. Soc. 2017, 96, 307-316. [CrossRef]

18. Alfuraidan, M.; Khamsi, M.A. A fixed point theorem for monotone asymptotically nonexpansive mappings. Proc. Am. Math. Soc. 2018, 146, 2451-2456. [CrossRef]

(C) 2019 by the authors. Licensee MDPI, Basel, Switzerland. This article is an open access article distributed under the terms and conditions of the Creative Commons Attribution (CC BY) license (http://creativecommons.org/licenses/by/4.0/). 